\title{
NEW PARAMETRIC SOLUTIONS OF SOME ABEL EQUATIONS
}

\author{
M. T. MUSTAFA
}

Received 15 May, 2014

\begin{abstract}
New parametric solutions of some Abel equations of second kind are found. The scheme for construction of these solutions utilizes a combination of differential invariants of a symmetry of an associated second order ODE and the solutions of the associated ODE.
\end{abstract}

2010 Mathematics Subject Classification: 34A05; 70G65

Keywords: Abel equations, first order differential equations, Lie symmetries, exact solutions

\section{INTRODUCTION}

Riccati and Abel equations form an interesting class of first order ODEs due to the difficulties involved in their integration as well as for the reason that the search for the solutions to many applied problems reduces to the question of solving one of these equations. A description of different approaches, in Section 0.1.4 of [8], to deal with Riccati equation

$$
\frac{d V}{d t}=f_{2}(t) V^{2}+f_{1}(t) V+f_{0}(t)
$$

includes conversion of (1.1) to a second order linear equation and utilizing its solution to obtain solution of Riccati equation. In Lie symmetry setting it can be viewed as follows:

- Consider the symmetry

$$
X=0 \frac{\partial}{\partial x}+y \frac{\partial}{\partial y}
$$

of a second order linear ODE

$$
a(x) \frac{d^{2} y}{d x^{2}}+b(x) \frac{d y}{d x}+c(x) y=0 .
$$

- Utilize the invariants $\frac{1}{y} \frac{d y}{d x}$ and $x$ of symmetry $X$ to get the transformations

$$
t=x, \quad V=\frac{1}{y} \frac{d y}{d x}
$$


to convert Riccati equation (1.1) to an ODE of the form (1.3), which may be easier to solve and hence can lead to solution of (1.1) via the transformations (1.4).

The purpose of this note is to investigate if an approach similar to above can be adapted to find new solutions of some Abel equations. The general form of an Abel equation of the second kind is

$$
(V+g(t)) \frac{d V}{d t}=f_{2}(t) V^{2}+f_{1}(t) V+f_{0}(t) .
$$

There is no general procedure to find solutions of these equations. However some integration strategies, a listing of known integrable cases and a description of integrable classes can be found, for example, in [3,5,6,8]. An approach of integrating Abel equations admitting nonlocal exponential symmetry is presented by Adam-Mahomed in [1]. It should be noted that most of the solutions given in [8, Section 1.3] are in parametric form. Here we focus on a specific type of Abel equations of second kind, not contained in [8, Section 1.3], and develop an integration strategy that leads to parametric solutions. Our approach involves computations using Lie symmetries; the reader is referred to $[2,4,7]$ for an introduction to the subject of symmetry analysis.

\section{INTEGRATION SCHEME AND EXAMPLES}

We consider Abel equations of the form

$$
(V+b t) \frac{d V}{d t}=a V+F(t), \quad a, b=\mathrm{constant}
$$

and ask the question:

Is there a symmetry $X$ of a second order $O D E$

$$
\frac{d^{2} y}{d x^{2}}=f\left(x, y, \frac{d y}{d x}\right)
$$

whose similarity variables provide a conversion relation between ODEs (2.1) and (2.2).

If such a symmetry is found and ODE (2.2) can be solved, it can be used to get solution of Abel equation (2.1) by utilizing the solution of second order ODE (2.2) via similarity variables of $X$.

As an ansatz for symmetry $X$ of ODE (2.2), we take a scaling symmetry of the form

$$
X=x \frac{\partial}{\partial x}+A y \frac{\partial}{\partial y}
$$

whose differential invariants are $\frac{y}{x^{A}}, x^{1-A} \frac{d y}{d x}$. The corresponding similarity variables

$$
t=\frac{y}{x^{A}}, \quad V=x^{1-A} \frac{d y}{d x}
$$


provide the transformations between ODEs (2.1) and (2.2) and convert ODE (2.1) to

$$
\frac{x^{2} \frac{d^{2} y}{d x^{2}}+x \frac{d y}{d x}-A x \frac{d y}{d x}}{x \frac{d y}{d x}-A y}=\frac{a x\left(\frac{d y}{d x}\right)+x^{A} F\left(y x^{-A}\right)}{x \frac{d y}{d x}+b y}
$$

which, upon choosing $A=-b$, reduces to

$$
x^{2} \frac{d^{2} y}{d x^{2}}+(b+1-a) x \frac{d y}{d x}-x^{-b} F\left(x^{b} y\right)=0
$$

In general, the values of undetermined parameters $a, b$ for which ODE (2.4) can be solved would lead to corresponding solvable cases of Abel equation (2.1). For the specific case $b=(a-1)$ we have

Lemma 1. An Abel equation of second kind of the form

$$
(V+(a-1) t) \frac{d V}{d t}=a V+F(t), \quad a=\mathrm{constant}
$$

transforms to a second order ODE of the form

$$
\frac{d^{2} y}{d x^{2}}=x^{-1-a} F\left(x^{a-1} y\right)
$$

through the similarity variables

$$
t=\frac{y}{x^{1-a}}, \quad V=x^{a} \frac{d y}{d x}
$$

of the symmetry

of ODE (2.6).

$$
X=x \frac{\partial}{\partial x}+(1-a) y \frac{\partial}{\partial y}
$$

An effective implementation of Lemma 1 requires a suitable choice of the value of $a$ for which ODE (2.6) is solved which will lead to parametric solutions of Abel equation (2.5) via the transformations (2.7). The scheme is implemented below to produce some new solutions of Abel equations.

Example 1. We consider the class of Abel equations

$$
(V+(a-1) t) \frac{d V}{d t}=a V+t^{n}, \quad n \neq 1 .
$$

By above Lemma, for $a=\frac{n+1}{n-1}$, it reduces to

$$
\frac{d^{2} y}{d x^{2}}=y^{n}
$$


via transformations

$$
t=y x^{\frac{2}{n-1}}, \quad V=x^{\frac{n+1}{n-1}} \frac{d y}{d x} .
$$

Case 1: For $n=2$, the ODE (2.9) can be solved to get solution

$$
y(x)=6 \text { Weierstrass } P\left(x+C_{1}, 0, C_{2}\right)
$$

where Weierstrass $P$ denotes the Weierstrass elliptic function. Hence we obtain, via (2.10), the parametric solution

$$
\begin{aligned}
t(x) & =6 x^{2} \text { Weierstrass } P\left(x+C_{1}, 0, C_{2}\right) \\
V(x) & =6 x^{3} \text { WeierstrassPPrime }\left(x+C_{1}, 0, C_{2}\right)
\end{aligned}
$$

of Abel equation (2.8) for $n=2$ and $a=3$.

Case 2: For $n=3$, the ODE (2.9) can be solved to get solution

$$
y(x)=C_{2} \operatorname{JacobiSN}\left(\left(1 / 2 i \sqrt{2} x+C_{1}\right) C_{2}, i\right)
$$

where JacobiSN denotes the Jacobi elliptic function. Hence we obtain, via (2.10), the parametric solution

$$
\begin{aligned}
t(x) & =x C_{2} \operatorname{JacobiSN}\left(\left(1 / 2 i \sqrt{2} x+C_{1}\right) C_{2}, i\right), \\
V(x) & =\frac{i}{2} x^{2} C_{2}^{2} \sqrt{2} \operatorname{JacobiCN}\left(\left(\frac{i}{2} \sqrt{2} x+C_{1}\right) C_{2}, i\right) \operatorname{JacobiDN}\left(\left(\frac{i}{2} \sqrt{2} x+C_{l}\right) C_{2}, i\right)
\end{aligned}
$$

of Abel equation (2.8) for $n=3$ and $a=2$.

Case 3: For $n=-3$, the ODE (2.9) can be solved to get solution

$$
y(x)=\frac{\sqrt{C_{1}\left(1+x^{2} C_{1}^{2}+2 x C_{1}^{2} C_{2}+C_{2}^{2} C_{1}^{2}\right)}}{C_{1}} .
$$

Hence we obtain, via (2.10), the parametric solution

$$
\begin{aligned}
t(x) & =\frac{\sqrt{C_{1}\left(1+x^{2} C_{1}^{2}+2 x C_{1}^{2} C_{2}+C_{2}^{2} C_{1}^{2}\right)}}{\sqrt{x} C_{l}}, \\
V(x) & =\frac{1}{2} \frac{\sqrt{x}\left(2 x C_{1}^{2}+2 C_{1}^{2} C_{2}\right)}{\sqrt{C_{l}\left(1+x^{2} C_{l}^{2}+2 x C_{1}^{2} C_{2}+C_{2}^{2} C_{l}^{2}\right)}}
\end{aligned}
$$

of Abel equation (2.8) for $n=-3$ and $a=\frac{1}{2}$.

Case 4: For $n=-1$, a first integral the ODE (2.9) is obtained as

$$
\frac{d y}{d x}= \pm \sqrt{2 \ln (y)}
$$


which can be integrated to get

$$
x=\frac{-i}{\sqrt{2}} \sqrt{\pi} \operatorname{erf}(i \sqrt{\ln (y)})
$$

where erf denotes the error function. Hence we obtain, via (2.10), the parametric solution

$$
\begin{aligned}
& t(y)= \pm \frac{\sqrt{2} i y}{\sqrt{\pi} \operatorname{erf}(i \sqrt{\ln (y)})}, \\
& V(y)= \pm \sqrt{2} \sqrt{\ln (y)}
\end{aligned}
$$

of Abel equation (2.8) for $n=-1$ and $a=0$.

Case 5: $n \neq-1$. For general $n$ with $n \neq-1$ a first integral the ODE (2.9) is obtained as

$$
\frac{d y}{d x}= \pm \sqrt{\frac{2}{n+1}} y^{\frac{n+1}{2}}
$$

which can be integrated for $n \neq 1$ to get

$$
x(y)=\mp \frac{\sqrt{2(n+1)}}{n-1} y^{-\frac{n-1}{2}}+C
$$

Hence the transformations (2.10) lead to the parametric solutions

$$
\begin{aligned}
& t(y)=\left(\mp \frac{\sqrt{2(n+1)}}{n-1}+C y^{\frac{n-1}{2}}\right)^{\frac{2}{n-1}} \\
& V(y)= \pm \frac{\sqrt{2}}{\sqrt{n+1}}\left(\mp \frac{\sqrt{2(n+1)}}{n-1}+C y^{\frac{n-1}{2}}\right)^{\frac{n+1}{n-1}}
\end{aligned}
$$

of Abel equation (2.8) for $n \neq \pm 1$ and $a=\frac{n+1}{n-1}$.

For Abel equations of the form (2.1) with $F(t)=\lambda t$ we obtain the following solutions.

Example 2. Consider the Abel equation

$$
(V+b t) \frac{d V}{d t}=a V+\lambda t, \quad a, b=\mathrm{constant}
$$

Then, as in equation (2.4), the similarity transformations

$$
t=\frac{y}{x^{-b}}, \quad V=x^{1+b} \frac{d y}{d x}
$$


transform (2.11) to

$$
x^{2} \frac{d^{2} y}{d x^{2}}+(b+1-a) x \frac{d y}{d x}-\lambda y=0
$$

which has the solution

$$
y(x)=C_{1} x^{\frac{a}{2}-\frac{b}{2}+\frac{1}{2} \sqrt{4 \lambda+a^{2}-2 a b+b^{2}}}+C_{2} x^{\frac{a}{2}-\frac{b}{2}-\frac{1}{2} \sqrt{4 \lambda+a^{2}-2 a b+b^{2}}} .
$$

This leads to, via (2.12), the parametric solution

$$
\begin{aligned}
t(x)= & C_{1} x^{\frac{a}{2}+\frac{b}{2}+\frac{1}{2} \sqrt{4 \lambda+a^{2}-2 a b+b^{2}}}+C_{2} x^{\frac{a}{2}+\frac{b}{2}-\frac{1}{2} \sqrt{4 \lambda+a^{2}-2 a b+b^{2}}}, \\
V(x)= & C_{1} x^{\frac{a}{2}+\frac{b}{2}+\frac{1}{2} \sqrt{4 \lambda+a^{2}-2 a b+b^{2}}}\left(\frac{a}{2}-\frac{b}{2}+\frac{1}{2} \sqrt{4 \lambda+a^{2}-2 a b+b^{2}}\right) \\
& +C_{2} x^{\frac{a}{2}+\frac{b}{2}-\frac{1}{2} \sqrt{4 \lambda+a^{2}-2 a b+b^{2}}}\left(\frac{a}{2}-\frac{b}{2}-\frac{1}{2} \sqrt{4 \lambda+a^{2}-2 a b+b^{2}}\right)
\end{aligned}
$$

of Abel equation (2.11). Viewing equation (2.13) as an eigenvalue problem, we determine that the eigenvalues $\lambda=n(n+b-a)$ and the eigenfunctions

$$
y(x)=C_{1} x^{n}+C_{2} x^{-n-b+a}
$$

satisfy equation (2.13). Hence, in addition to above solutions, the transformations (2.12) provide the parametric solutions

$$
\begin{aligned}
t(x) & =C_{1} x^{n+b}+C_{2} x^{-n+a}, \\
V(x) & =C_{1} x^{n+b} n+C_{2} x^{-n+a}(-n-b+a)
\end{aligned}
$$

of Abel equation (2.11) for $\lambda=n(n+b-a)$.

Though we have restricted to a specific class of Abel equations of the form (2.1) the strategy may be adapted for finding solutions of other types, as demonstrated in next example.

Example 3. We consider an Abel equation of second kind given by

$$
\left(-2 t^{2} V+t\right) \frac{d V}{d t}=V+2 t^{3}
$$

and take as ansatz for symmetry $X$ as

$$
X=x \frac{\partial}{\partial x}+A y \frac{\partial}{\partial y}
$$

whose differential invariants give similarity transformations

$$
t=\frac{x^{A}}{y}, \quad V=x^{1-A} \frac{d y}{d x},
$$


which transform Abel equation (2.14) to second order ODE

$$
\frac{y^{2}\left(-x^{2-2 A} \frac{d^{2} y}{d x^{2}}-x^{1-2 A} \frac{d y}{d x}+A x^{1-2 A} \frac{d y}{d x}\right)}{x \frac{d y}{d x}-A y}=\frac{x^{1-2 A} y^{3} \frac{d y}{d x}+2 x^{2 A}}{-2 y\left(x \frac{d y}{d x}-\frac{y}{2}\right)}
$$

Choosing $A=\frac{1}{2}$ simplifies above equation to

which has the solution

$$
\frac{d^{2} y}{d x^{2}}=y^{-3}
$$

$$
y(x)=\frac{\sqrt{C_{1}\left(1+x^{2} C_{1}^{2}+2 x C_{1}^{2} C_{2}+C_{2}^{2} C_{1}^{2}\right)}}{C_{1}} .
$$

So the similarity transformations

$$
t=\frac{\sqrt{x}}{y}, \quad V=\sqrt{x} \frac{d y}{d x}
$$

give the parametric solution

$$
\begin{aligned}
t(x) & =\frac{\sqrt{x} C_{1}}{\sqrt{C_{1}\left(1+x^{2} C_{1}^{2}+2 x C_{1}^{2} C_{2}+C_{2}^{2} C_{1}^{2}\right)}}, \\
V(x) & =\frac{1}{2} \frac{\sqrt{x}\left(2 x C_{1}^{2}+2 C_{1}^{2} C_{2}\right)}{\sqrt{C_{1}\left(1+x^{2} C_{1}^{2}+2 x C_{1}^{2} C_{2}+C_{2}^{2} C_{1}^{2}\right)}}
\end{aligned}
$$

of the Abel equation (2.14).

\section{CONCLUSION}

A new integration strategy for solving Abel equations of second kind is presented. Utilizing the differential invariants of Lie symmetries of an associated second order ODE, this strategy leads to parametric solutions of Abel equations. An implementation of the scheme is carried out to construct many new parametric solutions of some Abel equations.

\section{REFERENCES}

[1] A. A. Adam and F. M. Mahomed, "Integration of ordinary differential equations via nonlocal symmetries," Nonlinear Dynam, vol. 30, pp. 267-275, 2002, doi: 10.1023/A:1020518129295.

[2] G. Bluman and S. Kumei, Symmetries and Differential Equaions. New York: Springer-Verlag, 1989.

[3] E. S. Cheb-Terrab and A. D. Roche, "Abel odes: Equivalence and integrable classes," Comput. Phys. Commun., vol. 130, pp. 204-231, 2000, doi: 10.1016/S0010-4655(00)00042-4.

[4] N. H. Ibragimov, Elementary Lie group analysis and ordinary differential equations. Chichester: John Wiley \& Sons, 1999. 
[5] E. Kamke, Differentialgleichungen: Lösungsmethoden und Lösungen. New York: Chelsea Publishing Co., 1959.

[6] G. M. Murphy, Ordinary Differential Equations and their Solutions. Princeton: Van Nostrand, 1960.

[7] P. J. Olver, Applications of Lie groups to differential equations. New York: Springer-Verlag, 1986.

[8] A. D. Polyanin and V. F. Zaitsev, Handbook of exact solutions for ordinary differential equations. Boca Raton-London: CRC Press, 2003.

\section{Author's address}

M. T. Mustafa

Qatar University, Department of Mathematics, Statistics and Physics, Doha 2713, Qatar

E-mail address: tahir.mustafa@qu.edu.qa 